\title{
Potencial de generación de biogás y energía eléctrica. Parte II: residuos sólidos urbanos
}

\section{Potential of Power Generation from Biogas. Part II: Municipal Solid Waste}

\author{
Vera-Romero Iván \\ Universidad de la Ciénega del Estado de Michoacán de Ocampo \\ Correo:ivanverar@gmail.com \\ Estrada-Jaramillo Melitón \\ Universidad de la Ciénega del Estado de Michoacán de Ocampo \\ Correo:mestrada@ucienegam.edu.mx
}

\author{
Martínez-Reyes José \\ Universidad de la Ciénega del Estado de Michoacán de Ocampo \\ Correo:jmartinez@ucienegam.edu.mx \\ Ortiz-Soriano Agustina \\ Universidad de la Ciénega del Estado de Michoacán de Ocampo \\ Correo:aortiz@ucienegam.edu.mx
}

Información del artículo: recibido: abril de 2014, aceptado: agosto de 2014

\section{Resumen}

El objetivo de este trabajo es estimar la cantidad de biogás que se podría obtener a través de la descomposición anaeróbica de la fracción orgánica de los residuos sólidos urbanos (RSU) dispuestos en un relleno sanitario, captándola y aprovechándola para generar energía eléctrica que pueda ser consumida por la región Ciénega de Chapala correspondiente al estado de Michoacán, México. Para la estimación de biogás capturado se empleó el Modelo Mexicano de Biogás versión 2.0, captando RSU durante 11 años con una vida útil del proyecto de 21 años. Para el análisis de generación de energía eléctrica se consideró un costo promedio de la tarifa 5-A para servicio público de la CFE. Se evaluaron cuatro escenarios posibles: óptimo, intermedio óptimo, intermedio pesimista y pesimista variando características como manejo de sitio adecuado, presencia de incendios, cobertura, lixiviados, entre otros. Cada uno de los escenarios justifica económicamente la construcción de un relleno sanitario intermunicipal obteniendo beneficios económicos sustanciales a largo plazo $\left(26.5 \times 10^{6}\right.$ USD, $22.8 \times 10^{6}, 17.9 \times 10^{6}$ y $11.7 \times 10^{6}$, respectivamente), al mismo tiempo que se contribuye a la mitigación del cambio climático y a la prevención de enfermedades.

\section{Descriptores:}

- biogás

- residuos sólidos urbanos

- relleno sanitario

- energía eléctrica 


\begin{abstract}
The objective of this work is to estimate the amount of biogas that could be obtained from the anaerobic decomposition of the organic fraction of the municipal solid waste (MSW) disposed in a sanitary landfill, by capturing and taking advantage of it to generate electricity which can be consumed by Ciénega Region of Chapala in the state of Michoacán, México. To estimate the biogas captured, the Mexican Model of Biogas version 2.0 was used; capturing MSW for 11 years with a project life of 21 years. For the analysis of power generation an average cost for schedule rate 5-A from the CFE for public service was used. Four possible scenarios were evaluated: optimal, intermediate optimal, intermediate pessimistic and pessimistic; varying characteristics such as adequate handling site, fire presence, coverage, leachate, among others. Each of the scenarios, economically justify the construction of an inter-municipal landfill obtaining substantial long-term economic benefits. $\left(26.5 \times 10^{6}\right.$ USD, $22.8 \times 10^{6}, 17.9 \times 10^{6}$ and $11.7 \times 10^{6}$ respectively), while contributing to climate change mitigation and prevention of diseases.
\end{abstract}

\section{Keywords:}

- biogas

- municipal solid waste

- landfill

- electric power

\section{Introducción}

México se encuentra situado entre los principales países generadores de metano derivado de rellenos sanitarios (USEPA, 2005; Johari et al., 2012) y está localizado dentro de los diez países más productores de residuos sólidos urbanos (RSU) a nivel mundial (Rawat y Ramanathan, 2011). Los tiraderos a cielo abierto en la República Mexicana son muy comunes, tan solo en el Estado de Michoacán 60\% de la disposición final de los RSU se realiza en sitios no controlados (www.inegi.org.mx). Todo esto significa un grave problema medio-ambiental de alto riesgo (Kumar et al., 2004; Laurila et al., 2005), que afecta el paisaje, la flora y fauna local, cuerpos de agua, así como la presencia latente de focos de contaminación que atentan contra la vida humana (Aguilar et al., 2009; Ayomoh et al., 2008, Moy et al; 2008; Gillett, 1992; Karak et al., 2013).

Para enfrentar este tipo de problemas, se recurre a diferentes formas para minimizar las posibles afecciones, tales como: el confinamiento, el reciclado de materiales, la incineración, la composta o la construcción de rellenos sanitarios inertes. En la región Ciénega perteneciente al Estado de Michoacán (figura 1) no se cuenta con información oficial de que se lleve a cabo alguna de estas prácticas ambientalistas.

La Región Ciénega está conformada por nueve municipios al noroeste del estado: Briseñas, Cojumatlán de Régules, Jiquilpan, Pajacuarán, Sahuayo, Venustiano Carranza, Villamar, Vista Hermosa e Ixtlán. Entre ellas generan una gran cantidad de RSU que se desechan en tiraderos a cielo abierto sin ningún tipo de control, exponiendo ganado, cultivos y población a vectores de contaminación altamente perjudiciales.
Una alternativa de solución para este problema es la creación de un relleno sanitario que sirva para confinar los RSU en un lugar seguro donde se evite la liberación de gases altamente contaminantes como el metano $\left(\mathrm{CH}_{4}\right)$, el cual es un gas de efecto invernadero (GEI) que se encuentra en la emanación de gases de los tiraderos de basura o rellenos sanitarios y se conoce como biogás; también se evitarían filtraciones de los lixiviados a los mantos freáticos que sirven para el riego de cultivos y para dar de beber al ganado, además de confinar residuos altamente carcinógenos (Gillett, 1992; Karak et al., 2013).

El biogás se produce a través de una serie de etapas que se presentan en ausencia de oxigeno (proceso anaeróbico). Dicho proceso pasa por la fase de hidrólisis en un principio, donde se encuentran las bacterias fermentativas, posteriormente se presenta la acidogénesis y la acetogénesis, para finalmente dar paso a la fase de metanogénesis donde se forma el metano (Temelis y Ulloa, 2007).

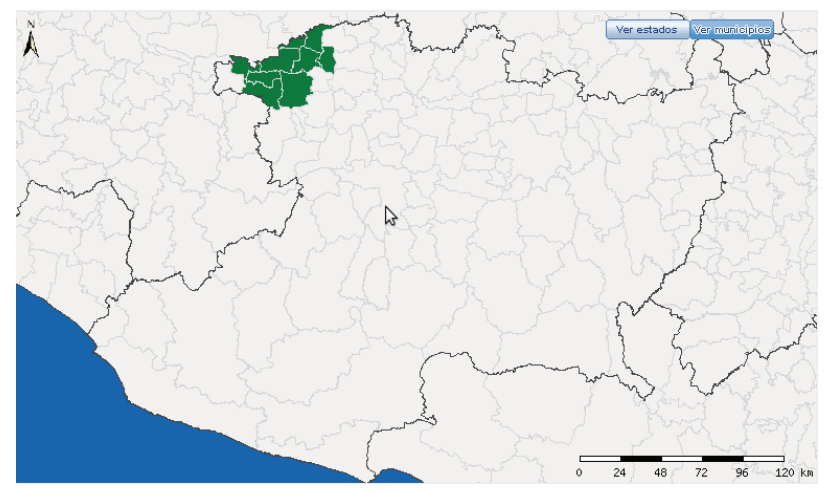

Figura 1. Región Ciénega 
Este biogás contiene aproximadamente $50 \%$ de $\mathrm{CH}_{4}$ (Aguilar et al., 2009), que cuando se captura en los rellenos sanitarios suele simplemente quemarse para reducir el grado de contaminación, ya que el $\mathrm{CH}_{4}$ tiene un potencial de calentamiento global 21 veces más que el $\mathrm{CO}_{2}$ (Batjes y Bridges, 1992). Sin embargo, de esta manera no se aprovecha su poder calorífico para producir algún tipo de beneficio extra, ya sea el de calentamiento o el de generar energía eléctrica a través de alguna máquina térmica (Stege y Davila, 2009; Aguilar et al., 2011), como el uso de un motor de combustión interna, la implementación de un ciclo Rankine o la incorporación de un ciclo Brayton.

En este trabajo se analiza el potencial de generación de energía eléctrica obtenida mediante el aprovechamiento de la energía contenida en el biogás que sería producido y capturado directamente de un relleno sanitario intermunicipal propuesto para la región Ciénega (Jaramillo, 1991; Bove y Lunghi, 2006). El estudio presenta cuatro escenarios posibles que tienen que ver finalmente con la eficiencia de captación de biogás, realizando un análisis de beneficios económicos (Murphy y McKeogh, 2004; Murphy et al., 2004) para cada uno de ellos y la estimación de la disminución del $\mathrm{CO}_{2}$ eq. Para estimar la generación de biogás en el relleno sanitario se empleó el Modelo Mexicano de Biogás versión 2.0 (Stege y Davila, 2009; Aguilar et al., 2011).

\section{Materiales y métodos}

Estimación de residuos per cápita

Para el modelado de generación de biogás en cada uno de los escenarios se requiere conocer: la concentración de los RSU, que para todos los casos se tomó la predefinida por el modelo (tabla 1), el valor de la densidad del $\mathrm{CH}_{4}\left(7.168 \times 10^{-04} \mathrm{t} \mathrm{m}^{-3}\right)$, la cantidad de RSU, que se pretende sea confinada en el relleno sanitario anualmente; para el caso propuesto, es la suma de todos los municipios que conforman la Región Ciénega (tabla 2).

Tabla 1. Caracterización predefinida de los RSU para Michoacán

\begin{tabular}{lc}
\hline Categoría de residuo & Datos específicos del sitio \% \\
\hline Comida & 34.50 \\
Papel y cartón & 8.10 \\
Poda (jardines) & 10.60 \\
Madera & 4.60 \\
Caucho, piel, huesos & 2.30 \\
y paja & 2.30 \\
Textiles & 10.50 \\
Otros orgánicos & 27.00 \\
Metales &
\end{tabular}

Tabla 2. Población y generación de RSU de la Región Ciénega

\begin{tabular}{lcc}
\hline \multicolumn{1}{c}{ Municipio } & Habitantes & RSU t año $^{-1}$ \\
\hline Briseñas & 10,653 & 2,693 \\
Cojumatlán de Régules & 9,980 & 2,523 \\
Jiquilpan & 34,199 & 8,646 \\
Pajacuarán & 19,450 & 4,917 \\
Sahuayo & 72,841 & 18,415 \\
Venustiano Carranza & 23,457 & 5,930 \\
Villamar & 16,991 & 4,296 \\
Vista Hermosa & 18,995 & 4,802 \\
Ixtlán & 13,584 & 3,434 \\
TOTAL & 220,150 & 55,657 \\
\hline
\end{tabular}

Debido a que no hay datos reportados de la producción de RSU para cada uno de los municipios considerados, se tomó el valor de la generación de residuos de todo el Estado (1,100,000 $\mathrm{t}$ año $^{-1}$, datos INEGI 2008), dividido entre el número total de habitantes $(4,351,037$ habs, Censo INEGI 2010), de esta manera se obtuvo un índice promedio aproximado per cápita $\left(0.693 \mathrm{~kg} \mathrm{hab}^{-1}\right.$ día $\left.^{-1}\right)$ que posteriormente fue afectado por el número de habitantes de toda la región (tabla 2). De esta manera se obtuvo el valor de los RSU totales que se introdujo al modelo.

\section{Estimación de la producción de biogás}

Para la estimación de producción de biogás se empleó el Modelo Mexicano de Biogás versión 2.0, desarrollado por SCS Engineers bajo acuerdo con el programa LMOP (Landfill Methane Outreach) de la Agencia de Protección Ambiental de Estados Unidos (USEPA). El modelo genera proyecciones de producción y captura de biogás dependiendo el manejo de los residuos y el arreglo del relleno sanitario, con la finalidad de realizar estudios de factibilidad a corto, mediano y largo plazo de este tipo de proyectos. Es posible observar, el potencial de beneficios que se pueden obtener al implementar un sitio de estos, realizar la captura y aprovechamiento del biogás como fuente de energía renovable.

El Modelo Mexicano de Biogás v.2.0, está basado en una ecuación de decaimiento de primer orden (ecuación 1).

$$
Q_{L F G}=\sum_{t=0}^{n} \sum_{j=0.1}^{1} 2 k L_{0}\left[\frac{M_{i}}{10}\right]\left(e^{-k t_{i j}}\right)(M C F)(F)
$$

donde

$Q_{L F G}=$ flujo de biogás máximo esperado

$i \quad=$ incremento en tiempo de 1 año

$n=$ año del cálculo menos el año inicial de disposiciónde residuos 
j = incremento de tiempo en 0.1 años

$M_{i} \quad=$ masa de residuos dispuestos en el año $i$

$t_{i j} \quad=$ edad de la sección $j$ de la masa $M_{i}$ dispuestas en el año $i$

$M C F=$ factor de corrección de metano, este valor depende de la profundidad y el tipo de relleno sanitario

$F=$ factor de ajuste por incendios. El modelo asume que el biogás está compuesto de $50 \%$ de $\mathrm{CH}_{4} \mathrm{y}$ $50 \%$ de $\mathrm{CO}_{2}$ con menos de $1 \%$ de otras trazas constituyentes

$k=$ índice de generación de metano y su valor depen de del contenido de humedad, la disponibilidad de nutrientes, el pH y la temperatura en el relleno sanitario

$L_{0}=$ generación potencial de metano, el modelo asig na valores a, $k$ y $L_{0}$ dependiendo de la zona cli mática donde se localice el sitio y la rapidez de degradación de los residuos (Stege y Davila, 2009).

\section{Descripción de los escenarios}

Para la realización de este modelado se establecieron cuatro casos que corresponden a cuatro posibles escenarios, haciendo variaciones en las características específicas del relleno sanitario como se puede observar en la tabla 3.
Otras consideraciones que se contemplaron en el modelado de la generación de biogás fueron las siguientes: relleno sanitario tipo zanja con una profundidad de $12 \mathrm{~m}$, recibirá durante 11 años RSU con un crecimiento en la disposición final de $2 \%$ anual, con un sistema de captura de $85 \%$ del total del sitio, el sistema de captura funcionará durante 20 años (Flores et al., 2008), iniciando la recuperación del biogás en el año 2016, un año después de la apertura del sitio.

Los beneficios que el relleno sanitario conllevaría al realizarse, se muestran en distintos ámbitos: de forma ambiental, al evitar la emanación de $\mathrm{CH}_{4}$ directo al ambiente y teniendo un lugar para disponer los RSU durante 11 años. De forma económica, aprovechar la energía contenida en el biogás y transformarla en energía eléctrica para la región, significaría un ahorro en la facturación con la Comisión Federal de Electricidad (CFE).

Para calcular la energía eléctrica producida en $\mathrm{kWh}$, se requiere conocer el flujo másico del biogás producido por año, el poder calorífico y una eficiencia del equipo térmico, lo cual viene dado en el modelo (Heat Rate de 10,800 BTU por $\mathrm{kWh}$ ), con factor de planta de $90 \%$. La energía producida queda afectada por el costo del $\mathrm{kWh}$ promedio reportado por CFE para una tarifa promedio 5-A (servicios públicos). El costo de la energía eléctrica queda afectada por un incremento progresivo de $6 \%$ anual en la facturación durante los años de vida del proyecto.

Tabla 3. Características de los escenarios propuestos

\begin{tabular}{|c|c|c|c|c|c|c|c|}
\hline \multirow[b]{2}{*}{ Escenarios } & \multirow[b]{2}{*}{$\begin{array}{l}\text { Manejo } \\
\text { del sitio }\end{array}$} & \multirow[b]{2}{*}{ Incendio } & \multirow{2}{*}{$\begin{array}{l}\text { Control en la } \\
\text { cubierta de los } \\
\text { residuos }\end{array}$} & \multirow[b]{2}{*}{$\begin{array}{c}\text { Compactación } \\
\text { adecuada }\end{array}$} & \multirow{2}{*}{$\begin{array}{l}\text { Disposición } \\
\text { adecuada de } \\
\text { residuos }\end{array}$} & \multicolumn{2}{|c|}{ Lixiviados } \\
\hline & & & & & & $\begin{array}{l}\text { Época de } \\
\text { lluvia }\end{array}$ & $\begin{array}{c}\text { Cualquier } \\
\text { época del año }\end{array}$ \\
\hline I óptimo & si & no & si & si & si & no & no \\
\hline $\begin{array}{l}\text { II intermedio } \\
\text { óptimo }\end{array}$ & si & no & si & si & si & si & no \\
\hline $\begin{array}{l}\text { III intermedio } \\
\text { pesimista }\end{array}$ & si & no & si & si & si & si & si \\
\hline IV pesimista & no & si & no & no & no & si & si \\
\hline
\end{tabular}

Tabla 4. Beneficios totales obtenidos

\begin{tabular}{lcccccc}
\hline \multicolumn{1}{c}{ Escenarios } & $\begin{array}{c}\text { Eficiencia } \\
\text { de captura } \\
\text { de biogás \% }\end{array}$ & $\begin{array}{c}\text { Reducción } \\
\text { de emisiones } \\
\text { totales RSU } \\
\mathrm{CO}_{2} \text { eq }\end{array}$ & $\begin{array}{c}\text { Reducción } \\
\text { de emisiones } \\
\text { totales RSU CH } \mathrm{H}_{4}\end{array}$ & $\begin{array}{c}\text { Ahorro por } \\
\text { Energía RSU } \\
\text { (USD) }\end{array}$ & $\begin{array}{c}\text { Ahorro por } \\
\text { Energía } \\
\text { Estiércol } \\
\text { (USD) }\end{array}$ & $\begin{array}{c}\text { Ahorro por } \\
\text { Energía } \\
\text { RSU+Estiércol } \\
\text { (USD) }\end{array}$ \\
\hline $\begin{array}{l}\text { I óptimo } \\
\begin{array}{l}\text { II intermedio } \\
\text { óptimo }\end{array}\end{array}$ & 71 & 360,479 & 17,166 & $26,550,790$ & $59,628,968$ & $86,179,758$ \\
$\begin{array}{l}\text { III intermedio } \\
\text { pesimista }\end{array}$ & 61 & 309,708 & 14,748 & $22,811,242$ & $59,628,968$ & $82,440,210$ \\
IV pesimista & 48 & 243,704 & 11,605 & $17,949,830$ & $59,628,968$ & $77,578,798$ \\
\hline
\end{tabular}


Al análisis de energía eléctrica y beneficios económicos se incorporó la potencia eléctrica que podría ofrecer la misma Región Ciénega a través de aprovechar el biogás generado por los estiércoles producidos por vacas y cerdos (Vera et al., 2014).

\section{Resultados y discusión}

Los beneficios totales obtenidos a lo largo de la vida útil del proyecto se pueden apreciar en la tabla 4 de concentrados y en el Apéndice (13.5 pesos por USD). El periodo contemplado para el estudio comprende de 2015 a 2035, empezando a aprovechar la captura de biogás al año de haber puesto en marcha el sitio.

Para estimar si un proyecto de este tipo es viable y factible técnica y económicamente, es necesario conocer el índice del costo del proyecto, que relaciona la inversión total y la cantidad de toneladas manejadas de RSU en el relleno al cabo de su vida útil. Existen variaciones de este índice dependiendo la región y el país, pudiendo no significar lo mismo para diferentes estudios de caso.

Al término del proyecto se dispondrá de 677,800 toneladas de RSU, en la tabla 5 se muestran los beneficios económicos por tonelada de basura, correspondiente al uso de biogás para generar energía eléctrica.

El beneficio obtenido por concepto de ahorro de energía eléctrica se comparó con el costo de un estudio que incluye tres importantes aspectos (Chong et al., 2005): costo de inversión, operación y mantenimiento, y clausura con un costo total de 8.89 USD t $^{-1}$. Como se puede observar, los cuatro escenarios estudiados se encuentran por encima del costo de un relleno sanitario, lo cual indica que es pre-factible un proyecto de estas características inclusive si la eficiencia de captura de biogás fuera la más baja $(40 \%)$.

Es importante considerar que los resultados obtenidos de los valores económicos son indicativos y pre- vios, para poder realizar estudios más a fondo es necesario desarrollar un análisis financiero que considere las variaciones de los costos de materiales de construcción, equipos, inflación, costo de los combustibles para generar la energía eléctrica, entre otros. Al mismo tiempo, es necesario caracterizar los RSU generados por los municipios.

Tabla 5. Beneficios por tonelada de desechos

\begin{tabular}{lc}
\hline \multicolumn{1}{c}{ Escenario } & $\begin{array}{c}\text { Ahorro de } \\
\text { Energía (USD } \\
\mathrm{t}^{-1} \text { ) }\end{array}$ \\
\hline Optimista & 39.17 \\
Intermedio Optimista & 33.65 \\
Intermedio Pesimista & 26.48 \\
Pesimista & 17.36 \\
\hline
\end{tabular}

\section{Conclusiones}

De acuerdo con los resultados obtenidos en la simulación, los cuatro escenarios son opciones viables y factibles para llevarse a cabo económicamente hablando, con un ahorro bruto promedio de 19,768,743 USD y un costo de 6,025,642 USD. Es necesario que las evaluaciones económicas se realicen mediante un análisis que tome en cuenta el valor del dinero en el tiempo para la toma de decisiones al respecto. Por otro lado, disminuiría la cantidad de emanaciones de GEI a la atmósfera, con un valor promedio de 268,413 toneladas de $\mathrm{CO}_{2} \mathrm{eq}$.

El modelo es capaz de proveer una visión general para realizar análisis de situaciones que puedan surgir en un análisis de estas características, durante el tiempo de vida útil de un proyecto de esta naturaleza, enmarcando los aspectos más importantes a tener en cuenta en la selección del diseño o de la operación del sitio de disposición. 


\section{Apéndice}

Tabla 6. Resultados corridas

\begin{tabular}{|c|c|c|c|c|c|c|c|c|c|c|}
\hline \multirow[b]{3}{*}{ Núm. } & \multirow[b]{3}{*}{ Año } & \multirow{3}{*}{$\begin{array}{l}\text { Costo } \mathrm{kWh} \\
\text { promedio 5-A } \\
\text { (pesos) }\end{array}$} & \multicolumn{8}{|c|}{ Escenarios } \\
\hline & & & I & II & III & IV & I & II & III & IV \\
\hline & & & \multicolumn{4}{|c|}{ Capacidad Máxima de la Planta de Electricidad RSU (MW) } & \multicolumn{4}{|c|}{ Generación de energía con $90 \%$ Factor de Planta RSU $(\mathrm{kWh})$} \\
\hline 0 & 2015 & 0.00 & 0.000 & 0.000 & 0.000 & 0.000 & 0.000 & 0.000 & 0.000 & 0.000 \\
\hline 1 & 2016 & 2.78 & 0.118 & 0.102 & 0.080 & 0.052 & 932,274 & 800,968 & 630,270 & 413,177 \\
\hline 2 & 2017 & 2.94 & 0.220 & 0.189 & 0.149 & 0.098 & $1,735,418$ & $1,490,993$ & $1,173,240$ & 769,124 \\
\hline 3 & 2018 & 3.12 & 0.309 & 0.265 & 0.209 & 0.137 & $2,433,508$ & $2,090,760$ & $1,645,188$ & $1,078,512$ \\
\hline 4 & 2019 & 3.31 & 0.387 & 0.332 & 0.261 & 0.171 & $3,047,660$ & $2,618,412$ & $2,060,390$ & $1,350,700$ \\
\hline 5 & 2020 & 3.50 & 0.456 & 0.392 & 0.308 & 0.202 & $3,593,318$ & $3,087,217$ & $2,429,285$ & $1,592,531$ \\
\hline 6 & 2021 & 3.71 & 0.518 & 0.445 & 0.350 & 0.230 & $4,082,970$ & $3,507,904$ & $2,760,318$ & $1,809,542$ \\
\hline 7 & 2022 & 3.94 & 0.574 & 0.493 & 0.388 & 0.254 & $4,526,724$ & $3,889,157$ & $3,060,321$ & $2,006,210$ \\
\hline 8 & 2023 & 4.17 & 0.626 & 0.538 & 0.423 & 0.277 & $4,934,444$ & $4,239,452$ & $3,335,962$ & $2,186,908$ \\
\hline 9 & 2024 & 4.42 & 0.674 & 0.579 & 0.456 & 0.299 & $5,312,507$ & $4,564,266$ & $3,591,554$ & $2,354,463$ \\
\hline 10 & 2025 & 4.69 & 0.719 & 0.617 & 0.486 & 0.319 & $5,666,093$ & $4,868,052$ & $3,830,598$ & $2,511,170$ \\
\hline 11 & 2026 & 4.97 & 0.761 & 0.654 & 0.514 & 0.337 & $5,999,416$ & $5,154,427$ & $4,055,943$ & $2,658,896$ \\
\hline 12 & 2027 & 5.27 & 0.654 & 0.562 & 0.442 & 0.290 & $5,157,675$ & $4,431,242$ & $3,486,879$ & $2,285,843$ \\
\hline 13 & 2028 & 5.59 & 0.566 & 0.486 & 0.383 & 0.251 & $4,463,444$ & $3,834,790$ & $3,017,540$ & $1,978,165$ \\
\hline 14 & 2029 & 5.92 & 0.493 & 0.424 & 0.333 & 0.219 & $3,888,922$ & $3,341,186$ & $2,629,130$ & $1,723,541$ \\
\hline 15 & 2030 & 6.28 & 0.433 & 0.372 & 0.293 & 0.192 & $3,411,686$ & $2,931,167$ & $2,306,492$ & $1,512,034$ \\
\hline 16 & 2031 & 6.65 & 0.382 & 0.328 & 0.258 & 0.169 & $3,013,643$ & $2,589,186$ & $2,037,392$ & $1,335,624$ \\
\hline 17 & 2032 & 7.05 & 0.340 & 0.292 & 0.230 & 0.151 & $2,680,178$ & $2,302,688$ & $1,811,951$ & $1,187,835$ \\
\hline 18 & 2033 & 7.47 & 0.304 & 0.261 & 0.206 & 0.135 & $2,399,482$ & $2,061,527$ & $1,622,185$ & $1,063,433$ \\
\hline 19 & 2034 & 7.92 & 0.274 & 0.236 & 0.185 & 0.122 & $2,162,001$ & $1,857,494$ & $1,461,634$ & 958,182 \\
\hline 20 & 2035 & 8.40 & 0.249 & 0.214 & 0.168 & 0.110 & $1,959,997$ & $1,683,941$ & $1,325,068$ & 868,656 \\
\hline
\end{tabular}

Tabla 6. Resultados corridas (continuación)

\begin{tabular}{|c|c|c|c|c|c|c|c|c|c|c|}
\hline \multirow{4}{*}{$\begin{array}{c}\text { Núm. } \\
0\end{array}$} & \multirow{4}{*}{$\begin{array}{l}\text { Año } \\
2015\end{array}$} & \multirow{4}{*}{$\begin{array}{c}\begin{array}{c}\text { Generación } \\
\text { de Energía- } \\
\text { Estiércoles } \\
(\mathrm{kWh})\end{array} \\
0.000\end{array}$} & \multicolumn{8}{|c|}{ Escenarios } \\
\hline & & & I & II & III & IV & I & II & III & IV \\
\hline & & & \multicolumn{4}{|c|}{ Generación Energía RSU+Estiércoles (kWh) } & \multicolumn{4}{|c|}{ Ahorro por concepto de Energía RSU ( $\left.\$ \mathrm{kWh}^{-1} \mathrm{año}^{-1}\right)$} \\
\hline & & & 0.000 & 0.000 & 0.000 & 0.000 & 0.000 & 0.000 & 0.000 & 0.000 \\
\hline 1 & 2016 & $7,884,000$ & $8,816,274$ & $8,684,968$ & $8,514,270$ & $8,297,177$ & $2,587,680$ & $2,223,218$ & $1,749,417$ & $1,146,840$ \\
\hline 2 & 2017 & $7,884,000$ & $9,619,418$ & $9,374,993$ & $9,057,240$ & $8,653,124$ & $5,105,950$ & $4,386,802$ & $3,451,910$ & $2,262,919$ \\
\hline 3 & 2018 & $7,884,000$ & $10,317,508$ & $9,974,760$ & $9,529,188$ & $8,962,512$ & $7,589,465$ & $6,520,527$ & $5,130,906$ & $3,363,594$ \\
\hline 4 & 2019 & $7,884,000$ & $10,931,660$ & $10,502,412$ & $9,944,390$ & $9,234,700$ & $10,075,133$ & $8,656,100$ & $6,811,357$ & $4,465,223$ \\
\hline 5 & 2020 & $7,884,000$ & $11,477,318$ & $10,971,217$ & $10,313,285$ & $9,476,531$ & $12,591,741$ & $10,818,256$ & $8,512,726$ & $5,580,565$ \\
\hline 6 & 2021 & $7,884,000$ & $11,966,970$ & $11,391,904$ & $10,644,318$ & $9,693,542$ & $15,166,041$ & $13,029,979$ & $10,253,098$ & $6,721,475$ \\
\hline 7 & 2022 & $7,884,000$ & $12,410,724$ & $11,773,157$ & $10,944,321$ & $9,890,210$ & $17,823,210$ & $15,312,899$ & $12,049,494$ & $7,899,113$ \\
\hline 8 & 2023 & $7,884,000$ & $12,818,444$ & $12,123,452$ & $11,219,962$ & $10,070,908$ & $20,594,249$ & $17,693,651$ & $13,922,873$ & $9,127,217$ \\
\hline 9 & 2024 & $7,884,000$ & $13,196,507$ & $12,448,266$ & $11,475,554$ & $10,238,463$ & $23,502,450$ & $20,192,245$ & $15,888,980$ & $10,416,109$ \\
\hline 10 & 2025 & $7,884,000$ & $13,550,093$ & $12,752,052$ & $11,714,598$ & $10,395,170$ & $26,570,714$ & $22,828,359$ & $17,963,299$ & $11,775,941$ \\
\hline 11 & 2026 & $7,884,000$ & $13,883,416$ & $13,038,427$ & $11,939,943$ & $10,542,896$ & $29,821,831$ & $25,621,573$ & $20,161,238$ & $13,216,811$ \\
\hline 12 & 2027 & $7,884,000$ & $13,041,675$ & $12,315,242$ & $11,370,879$ & $10,169,843$ & $27,175,977$ & $23,348,375$ & $18,372,492$ & $12,044,189$ \\
\hline 13 & 2028 & $7,884,000$ & $12,347,444$ & $11,718,790$ & $10,901,540$ & $9,862,165$ & $24,929,133$ & $21,417,988$ & $16,853,498$ & $11,048,405$ \\
\hline 14 & 2029 & $7,884,000$ & $11,772,922$ & $11,225,186$ & $10,513,130$ & $9,607,541$ & $23,023,543$ & $19,780,790$ & $15,565,212$ & $10,203,861$ \\
\hline 15 & 2030 & $7,884,000$ & $11,295,686$ & $10,815,167$ & $10,190,492$ & $9,396,034$ & $21,410,060$ & $18,394,559$ & $14,474,407$ & $9,488,778$ \\
\hline 16 & 2031 & $7,884,000$ & $10,897,643$ & $10,473,186$ & $9,921,392$ & $9,219,624$ & $20,046,863$ & $17,223,361$ & $13,552,809$ & $8,884,619$ \\
\hline 17 & 2032 & $7,884,000$ & $10,564,178$ & $10,186,688$ & $9,695,951$ & $9,071,835$ & $18,898,364$ & $16,236,622$ & $12,776,359$ & $8,375,613$ \\
\hline 18 & 2033 & $7,884,000$ & $10,283,482$ & $9,945,527$ & $9,506,185$ & $8,947,433$ & $17,934,280$ & $15,408,325$ & $12,124,584$ & $7,948,338$ \\
\hline 19 & 2034 & $7,884,000$ & $10,046,001$ & $9,741,494$ & $9,345,634$ & $8,842,182$ & $17,128,847$ & $14,716,333$ & $11,580,065$ & $7,591,376$ \\
\hline 20 & 2035 & $7,884,000$ & $9,843,997$ & $9,567,941$ & $9,209,068$ & $8,752,656$ & $16,460,140$ & $14,141,810$ & $11,127,982$ & $7,295,010$ \\
\hline
\end{tabular}




\section{Referencias}

Aguilar-Virgen Q., Armijo-De Vega C., Taboada-González P. El potencial energético de los residuos sólidos municipales. Ingeniería Revista Académica, volumen 13, 2009: 59-62.

Aguilar-Virgen Q., Armijo-De Vega C., Taboada-González P. Modelo mexicano para la estimación de la generación de biogás. Ingeniería Revista Académica, volumen 15, 2011: 37-45.

Ayomoh M.K.O., Oke S.A., Adedji W.O., Charles-Owaba O.E. An approach to tackling the environmental and health impacts of municipal solid waste disposal in developing countries. Journal of Environmental Management, volumen 88, 2008: 108-114.

Bove R., Lunghi P. Electric power generation from landfill gas using traditional and innovative technologies. Energy Conversion and Management, volumen 47, 2006: 1391-1401.

Batjes N.H., Bridges E.M. World inventory of soil emissions. International soil reference and information centre, 1992, pp. 11-35.

Chong T.L., Matsufuji Y., Hassan M.N. Implementation of the semi-aerobic landfill system (Fukuoka method) in Developing Countries: A Malaysia Cost Analysis. Waste Management, volumen 25, 2005: 702-711.

Flores R., Muñoz-Ledo R., Flores B.B., Cano K.I. Estimación de la generación de energía a partir de biomasa para proyectos del programa de mecanismo de desarrollo limpio. Revista Mexicana de Ingeniería Química, volumen 7, 2008: 35-39.

Gillett J.W. Issues in risk assessment of compost from municipal solid waste: occupational health and safety, public health, and environmental concerns. Biogas and Bioenergy, volumen 3, 1992: 145-162.

Jaramillo J. Guía para el diseño, construcción y operación de rellenos sanitarios manuales, Washington,1991.

Johari A., Ahmed S.I., Hashim H., Alkali H., Ramli M. Economic and environmental benefits of landfill gas from municipal solid waste in Malaysia. Renewable and Sustainable Energy Reviews, volumen 16, 2012: 2907-2912.
Karak T., Bhattacharyya P., Das T. Non-segregated municipal solid waste in an open dumping ground: A potential contaminant in relation to environmental health. International Journal of Environmental Science and Technology, volumen 10, 2013: 503-518.

Kumar S., Gaikwad S.A., Shekdar A.V., Kshirsagar P.S., Singh R.N. Estimation method for National Methane Emission from solid waste landfill. Atmospheric Environment, volumen 38, 2004: 3481-3487.

Laurila T., Tuovinen J.P., Lohila A., Hatakka J., Aurela M., Thum T., Pihlatie M., Rinne J., Vesala T. Measuring methane emissions from a landfill using a cost-effective micrometeorological method. GeophysicalResearch Letters, volumen 32, 2005 [en línea]. Disponible en: http://dx.doi.org/10.1029/2005GL023462

Moy P., Krishnan N., Ulloa P., Cohen S., Brandtl-Raul P.W. Options for management of municipal solid waste in New York City: A preliminary comparison of health risks and policy implications. Journal of Environmental Management, volumen 87, 2008: 73-79.

Murphy J.D., McKeogh E. Technical, economic and environmental analysis of energy production from municipal solid waste. Renewable energy, volumen 29, 2004: 1043-1057.

Murphy J.D., McKeogh E., Kiely G. Technical/economic/environmental analysis of biogas utilization. Applied Energy, volumen 77, 2004: 407-427.

Rawat M., Ramanathan A.L. Assessment of methane flux from municipal solid waste (MSW) landfill areas of Delhi, India. Journal of Environmental Protection, volumen 2, 2011: 399407.

Stege G.A., Davila J.L. Manual del usuario modelo mexicano de biogás versión 2.0. 2009: Washington DC.

Temelis N.J., Ulloa P.A. Methane generation in landfills. Renewable Energy, volumen 32, 2007: 1243-1257.

US-EPA. Global anthropogenic Non- $\mathrm{CO}_{2}$ greenhouse gas emissions: 1990-2020. U.S. EPA, 2005, Washington DC. 


\section{Este artículo se cita: \\ Citación estilo Chicago \\ Vera-Romero, Iván, Melitón Estrada-Jaramillo, José Martínez- Reyes, Agustina Ortiz-Soriano. Potencial de generación de bio- gás y energía eléctrica. Parte II: Residuos sólidos urbanos. Ingeniería Investigación y Tecnología, XVI, 03 (2015): 471-478. \\ Citación estilo ISO 690 \\ Vera-Romero I., Estrada-Jaramillo M., Martínez-Reyes J., Ortiz- Soriano A. Potencial de generación de biogás y energía eléctri- ca. Parte II: Residuos sólidos urbanos. Ingeniería Investigación y Tecnología, volumen XVI (número 3), julio-septiembre 2015: 471-478.}

\section{Semblanzas de los autores}

Iván Vera-Romero. Realizó estudios de licenciatura en el Instituto Tecnológico de Orizaba (ITO), obteniendo el grado de ingeniero mecánico en 2001. Obtuvo el grado de maestro en energía, sistemas energéticos: Procesos y uso eficiente de la energía por la Universidad Nacional Autónoma de México en 2011. Actualmente labora como profesor-investigador de la Universidad de la Ciénega del Estado de Michoacán de Ocampo.

Melitón Estrada-Jaramillo. Realizó los estudios de licenciatura en la Universidad Michoacana de San Nicolás de Hidalgo (UMSNH), obteniendo el grado de ingeniero mecánico en 2002. Obtuvo el grado de maestro en ciencias en ingeniería mecánica en el área de termofluidos: Sistemas de postcombustión para la disminución de contaminantes, por la Universidad Michoacana de San Nicolás de Hidalgo en 2004. Actualmente labora como profesor-investigador de la Universidad de la Ciénega del Estado de Michoacán de Ocampo y es coordinador de la trayectoria de ingeniería en energía.

José Martínez-Reyes. Realizó la licenciatura en el Instituto Politécnico Nacional (IPN), obteniendo el grado de ingeniero químico industrial en 1990. Obtuvo el grado de maestro en tecnología avanzada por el Instituto Politécnico Nacional en 2007 y el grado de doctor en ciencias de la tierra en 2013. Actualmente labora como profesorinvestigador de la Universidad de la Ciénega del Estado de Michoacán de Ocampo.

Agustina Ortiz-Soriano. Realizó los estudios de licenciatura en filosofía en la Universidad Autónoma Metropolitana (UAM). Obtuvo el grado de maestra en ciencias de la educación por el Instituto Superior de Ciencias de la Educación del Estado de México en 2009 y el grado de maestra en humanidades (filosofía política) en la UAM en 2010. Actualmente labora como profesor-investigador de tiempo completo en la Universidad de la Ciénega del Estado de Michoacán de Ocampo. 